\title{
1) Takayasu-Aortitis mit Befall der Pulmonalarterien - Differenzialdiagnose zur Lungenembolie
}

Beim Morbus Takayasu handelt es sich um eine chronisch entzündliche Gefäßerkrankung unklarer Ätiologie. In erster Linie sind dabei Arterien vom elastischen Typ wie die Aorta und ihre Äste sowie die Pulmonalarterien betroffen (Ferretti et al., Eur Radiol 1996; 6: 429-432). Histopathologisch zeigt sich eine granulo- matöse Entzündung, die von der Intima zur Adventitia fortschreitet und zu einer Fibrosklerose führt. Im Spätstadium kann es sowohl zu segmentalen Stenosen und Verschlüssen als auch zu Dilatationen und Aneurysmen kommen (Matsunaga et al., Radiographics 1997; 17: $\quad 579-594) . \quad$ Kontrastmittel-ver- 
stärkte Computer-Tomographie (CT) und Magnetresonanz-Tomographie (MRT) zeigen im Frühstadium der Erkrankung in der Regel eine deutliche Kontrastmittel (KM)-Aufnahme der verdickten Gefäßwand. Am besten kommen die Veränderungen im Spätstadium zur Darstellung. Wir berichten über eine Patientin, bei der nach auffälliger Lungenperfusionsszintigraphie die anschließend durchgeführte CT, MRT und Positronen-Emissions-Tomographie (PET) zur Diagnose einer Takayasu-Aortitis mit Befall der linken Pulmonalarterie führten.

\section{Fallbeschreibung}

Eine 57-jährige bisher gesunde Patientin wurde wegen Dyspnoe und auskultierbaren Strömungsgeräuschen über der oberen Thoraxapertur mit der Verdachtsdiagnose einer Lungenembolie zur Perfusions- und Ventilationsszintigraphie überwiesen. Die körperliche Untersuchung ergab neben einer asymptomatischen Pulslosigkeit des rechten Armes keine weiteren klinisch relevanten Befunde. Die Sauerstoffsättigung und der Sauerstoff-Partialdruck $\left(\mathrm{pO}_{2}\right)$ im Blut waren normal. Die Laborwerte zeigten außer mäßig erhöhten Werten für BSG und CRP keine spezifischen Veränderungen. Die Röntgen-Thorax-Aufnahme im p.a. und seitlichen Strahlengang war mit Ausnahme einer diskreten globalen Kardiomegalie unauffällig. Die Szintigraphie ergab bei regelrechter Belüftung $\left({ }^{127} \mathrm{Xe}\right)$ eine homogene Minderperfusion (99mTc-MAA) der linken Lunge ohne segmentale Ausfälle (R/L-Ratio 3,65), vereinbar mit einer Einengung der linken

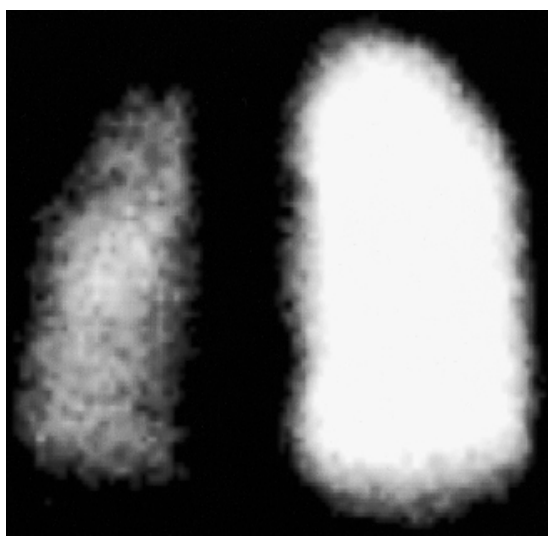

Abb. 1 Die Lungenperfusionsszintigraphie mit ${ }^{99 m}$ Tc (dorsale Ansicht) offenbart eine deutlich reduzierte Perfusion der linken Lunge ohne segmentale oder subsegmentale Ausfälle.
Pulmonalarterie (Abb.1). Die anschließend durchgeführte CT des Thorax (Somatom Plus 4, Siemens, Erlangen; Kollimation $3 \mathrm{~mm}$; Pitch 0,5; $240 \mathrm{~mA}$; $120 \mathrm{kV}$ ) mit jodhaltigem KM (Iopromid (Ultravist ${ }^{\circledR}$ Schering, Berlin), $120 \mathrm{ml} @$ $3,0 \mathrm{ml} / \mathrm{s}$ ) konnte eine zentrale Lungenembolie ausschließen, zeigte jedoch eine KM-Aufnahme der Gefäßwand der Aorta ascendens sowie des Truncus pulmonalis und der proximalen Pulmonalarterien (Abb. 2). Das Lumen der linken Pulmo-

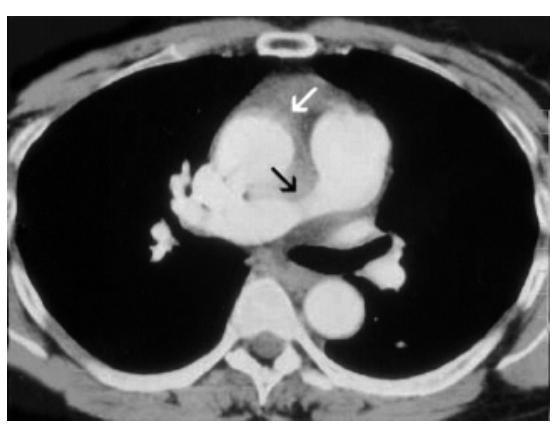

Abb. 2 Die kontrastmittelunterstützte CT weist eine entzündlich verdickte Wand der Aorta (weißer Pfeil) sowie eine umschriebene Stenose der linken A. pulmonalis (schwarzer Pfeil) mit geringer poststenotischer Dilatation auf.

nalarterie war leichtgradig eingeengt. Einzelne reaktiv vergrößerte mediastinale Lymphknoten waren abgrenzbar. Die daraufhin durchgeführte KM-verstärkte MRT/MRA (1,5 T, Signa Echo Speed, GE, Milwaukee, USA; 3D-GRE: TR/TE 4,0/1,9 ms; FoV 32.32; Matrix 192.256; Gd-DTPA (Magnevist ${ }^{\circledR}$ Schering, Berlin), 0,2 mmol/kg KG @ 2,0 ml/s) konnte diese Befunde bestätigen, wobei die Kontrastmittelanreicherung der Gefäßwände deutlich zur Darstellung kam. Zusätzlich zeigte die MRA Verengungen im Abgangsbereich der proximalen supraaortalen Gefäße, insbesondere eine hochgradige Stenose des Truncus brachiozephalicus, die ursächlich für die klinische Pulslosigkeit war (Abb.3). Die anschließend durchgeführte PET (Abb.4) mit [ $\left.{ }^{18} \mathrm{~F}\right]-F D G$ konnte eine umschriebene Anreicherung in Aorta und Truncus pulmonalis nachweisen. Die Kombination von klinischer Symptomatik, körperlichen Untersuchungsbefunden und Laborwerten mit den Ergebnissen der Bildgebung führte zur Diagnose eines M. Takayasu. Die daraufhin begonnene Therapie mit Steroiden und Methotrexat führte langfristig bei der Patientin zu einer klinischen Besserung: Die zuletzt durchgeführte Perfu-

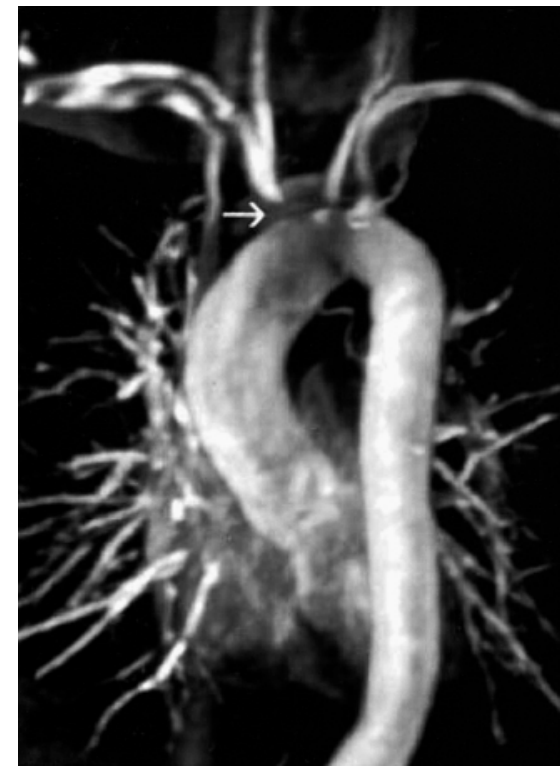

Abb. 3 Die kontrastmittelverstärkte MRA zeigt multiple Verengungen der supraaortalen Gefäße mit hochgradiger Stenose des Truncus brachiocephalicus. Das pulmonale Gefäßbett auf der linken Seite ist weniger kontrastiert.

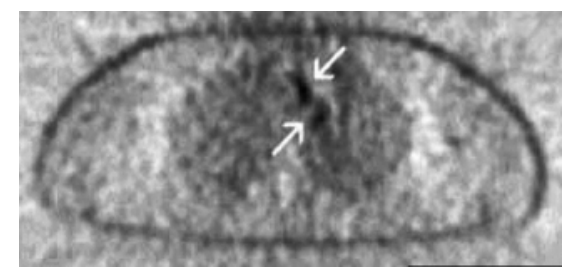

Abb. 4 Die PET mit $\left[{ }^{18} \mathrm{~F}\right]-\mathrm{FDG}$ weist eine Mehraufnahme der großen Gefäße als Korrelat der entzündlichen Beteiligung von Aorta und Truncus pulmonalis auf.

sionsszintigraphie der Lunge ergab eine Befundbesserung (R/L-Ratio 2,03), die in der MRA dokumentierten Stenosen stellten sich unverändert dar.

\section{Diskussion}

Die größte Prävalenz der Takayasu-Aortitis wird für heranwachsende Mädchen und junge Frauen angegeben. Obwohl eine Häufung im asiatischen Erdteil beobachtet wird, scheint die TakayasuAortitis weder geographisch noch ethnisch begrenzt zu sein (Rizzi et al., Int J Clin Lab Res 1999; 29: 8-13). Die Takayasu-Aortitis ist eine Systemerkrankung mit Allgemein- und Lokalsymptomen. $\mathrm{Zu}$ den Allgemeinsymptomen, welche in der Regel im Frühstadium vorkommen, zählen Unwohlsein, Fieber, Nachtschweiß, Arthralgien, Anorexie und Gewichtsverlust. Diese Symptome können Monate vor dem histopathologisch nachweisbaren Gefäßbefall auftre- 
ten. Im weiteren Verlauf führt die Gefäßentzündung zu Stenosen oder Aneurysmen der betroffenen Arterien mit Schmerzen im Versorgungsgebiet. Im Spätstadium kommt es zu arteriellen Verschlüssen mit manifesten Ischämien der betroffenen Regionen. Häufig tritt bei Befall der Arteria subclavia eine Pulslosigkeit des Armes der jeweiligen Seite auf. Der klinische Verlauf ist unterschiedlich und reicht von fulminant bis zu langsam progredient oder stationär; auch spontane Remissionen sind möglich. Die Komplikationen stehen in $\mathrm{Zu}-$ sammenhang mit der Lokalisation der betroffenen Gefäße. Die Therapie mit Immunsuppressiva kann den Krankheitsverlauf positiv beeinflussen und gelegentlich zur Remission führen (Churg, Clin Exp Immunol 1993, Suppl $1 ; 11-12)$.

Lupi beschreibt beim M. Takayasu 4 Typen in Abhängigkeit von der Verteilung des Gefäßbefalls, Typ 1: Aortenbogen mit brachiozephalen Gefäßen (Aortenbogensyndrom); Typ II: Befall der Aorta abdominalis mit den abdominalen Aortenästen und Beckenstammgefäßen; Typ III: kombinierter Befall von thorakaler und abdominaler Aorta und ihrer Aufteilungen; Typ IV: zusätzlicher Befall der Pulmonalarterien (Lupi et al., Chest 1975; 67: 69-74). Obwohl die Pulmonalarterien relativ häufig betroffen sind (50-86\%), wie größere, angiographisch untersuchte Patientenkollektive mit bekannter Takayasu-Aortitis gezeigt haben, ist ein Befall der Pulmonalarterien im Frühstadium selten (Hayashi et al., Radiology 1986; 159: $401-403$ ). Eine frühzeitige Diagnose ist jedoch wichtig, da der rechtzeitige Beginn einer immunsupprimierenden Therapie das Fortschreiten der Erkrankung im Gefäßbett der Lunge mit drohender pulmonaler Hypertonie aufhalten kann. Die klinischen Symptome eines Befalls der Aorta verdecken oftmals die Beteiligung der Pulmonalarterien. Zusätzlich sind die klinischen Zeichen und Charakteristika einer pulmonalen Arteriitis unspezifisch, die Mehrzahl der Patienten ist initial oft asymptomatisch. Im Verlauf entwickeln sich thorakales Engegefühl, chronische Dyspnoe sowie gelegentlich Pleuraergüsse und Hämoptysen (Koyabu et al., Chest 1993; 104: $1905-1906)$, so dass eine Lungenembolie differenzialdiagnostisch in Erwägung gezogen werden muss. Mittels CT und MRT/MRA ist es möglich, frühzeitig den pulmonalen Befall bei M.Takayasu von einer Lungen- embolie zu differenzieren. Dabei sind das charakteristische Wandenhancement und die damit verbundene Verengung des Lumens sowie entzündliche Veränderungen des mediastinalen Fettgewebes die wegweisenden Befunde in der CT und MRT bei der Abgrenzung zur Lungenembolie (Matsunaga et al., J Magn Reson Imaging 1998; 8: 406 - 414). Hierbei ist besonders die Wertigkeit der MRT hervorzuheben, die gerade bei jungen Patienten ohne die Anwendung von ionisierender Strahlung valide Ergebnisse liefert. Zusätzlich können mit der 3D-MRA entzündliche Gefäßveränderungen in multiplen virtuellen Ebenen beurteilt werden.

Die in dem hier vorgestellten Fall primär durchgeführte Lungenperfusionsszintigraphie zeigte eine Minderperfusion der linken Lunge als Folge der Wandveränderungen der linken $\mathrm{A}$. pulmonalis. Die Verdachtsdiagnose Lungenembolie war mittels Szintigraphie nicht auszuschließen. In der anschließend durchgeführten Kontrastmittel-unterstützen CT konnten jedoch die charakteristischen Befunde eines M. Takayasu mit Wandenhancement der Aorta ascendens sowie des Truncus pulmonalis und der linken Pulmonalarterie nachgewiesen werden. Die KM-verstärkte Akquisition einer 3D-MRA erfasste neben der Verengung der linken A. pulmonalis zusätzlich fokale Stenosen der supraaortalen Äste, unter anderem am Truncus brachiocephalicus als Korrelat zur Pulslosigkeit des rechten Armes. Die entzündliche Infiltration der großen Gefäße konnte darüber hinaus mittels $\left[{ }^{18} \mathrm{~F}\right]$ FDG-PET bestätigt werden. Zusammenfassend sollte unter den o.g. Umständen bei Verdacht auf zentrale Lungenembolie mit szintigrapischem Nachweis einer deutlich reduzierten Perfusion eine Takayasu-Aortitis Typ IV differenzialdiagnostisch in Erwägung gezogen werden, die mittels KM-verstärkter CT und/oder MRT sicher evaluiert werden kann.

Springende Punkte:

- M.Takayasu kann die Pulmonalarterien befallen (Typ IV nach Lupi)

- Lungenperfusionsszintigraphie kann bei M.Takayasu einen Perfusionsausfall zeigen

- CT und/oder MRT ermöglichen die Differenzierung zwischen M.Takayasu Typ IV und Lungenembolie.

S. Kneifel, T. A. G. M. Huismann, Zürich

\section{Erratum}

In Ausgabe 10/2001 der RöFo (Fortschr Röntgenstr 2001; 173: 960) wurde in der Arbeit von C. U. Herborn et al: „Takayasu-Aortitis mit Befall der Pulmonalarterien - Differenzialdiagnose zur Lungenembolie“ der Erstautor, Dr. C. U. Herborn nicht aufgeführt. Die korrekte Autorenliste lautet: C. U. Herborn, Essen; S. Kneifel, T. A. G. M. Huismann, Zürich. Wir bitten, diesen Fehler zu entschuldigen. 